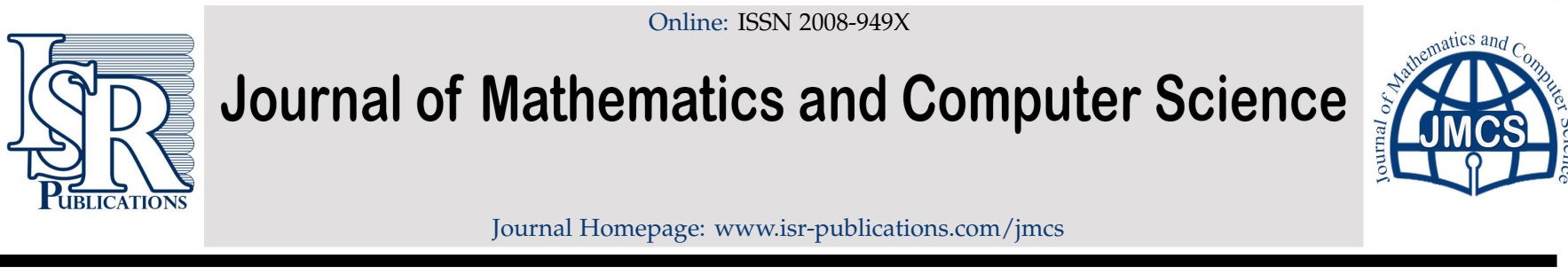

\title{
Best proximity points of admissible almost generalized weakly contractive mappings with rational expressions on b-metric spaces
}

\author{
Lakshmi Narayan Mishra $^{\mathrm{a}}$, Vinita Dewangan ${ }^{\mathrm{b}}$, Vishnu Narayan Mishra ${ }^{\mathrm{c}, *}$, Seda Karateke $^{\mathrm{d}}$ \\ ${ }^{a}$ Department of Mathematics, School of Advanced Sciences, Vellore Institute of Technology (VIT) University, Vellore 632014 , Tamil \\ Nadu, India. \\ ${ }^{b}$ Govt. S.N. College Nagari Distt.-Dhamtari, Chhattishgarh, India. \\ ${ }^{c}$ Dept. of Mathematics, Indira Gandhi National Tribal University, Lalpur, Amarkantak, Anuppur, Madhya Pradesh 484 887, India. \\ ${ }^{d}$ Department of Mathematics and Computer Science, Faculty of Science and Letters, Istanbul Arel University, Istanbul-34537, Turkey.
}

\begin{abstract}
The aim of this paper is to introduce almost generalized proximal $(\alpha-\psi-\varphi-\theta)$-weakly contractive mappings with rational expressions and prove the best proximity point theorems for such mappings. The main results of this paper are generalizations of several comparable results in the literature.
\end{abstract}

Keywords: Fixed point, best proximity points, metric space, b-metric space, admissible mapping.

2020 MSC: 41A65, 47H05, 47H09, 47H10, 54H25, 90С30.

(C)2021 All rights reserved.

\section{Introduction}

The existence and uniqueness of a fixed point of non-self mappings is one of the interesting subjects in fixed point theory. In fact, given nonempty closed subsets $A$ and $B$ of a complete metric space $(X, d)$, a contraction non-self-mapping $T: A \rightarrow B$ does not necessarily yield a fixed point $T x=x$. In this case, it is very natural to investigate whether there is an element $x$ such that $d(x, T x)$ is minimum. A notion of best proximity point appears at this point. Let $(X, d)$ is a metric space, and $A, B$ are subsets of $X$. A point $x$ is called best proximity point of $T: A \rightarrow B$ if $d(x, T x)=d(A, B)$, where $d(A, B)=\inf \{d(x, y): x \in A, y \in B\}$.

$A$ best proximity point represents an optimal approximate solution to the equation $T x=x$ whenever a non-self-mapping $T$ has no fixed point. It is clear that a fixed point coincides with a best proximity point if $d(A, B)=0$. Since a best proximity point reduces to a fixed point if the underlying mapping is assumed to be self-mappings, the best proximity point theorems are natural generalizations of the Banach's contraction principle.

\footnotetext{
${ }^{*}$ Corresponding author

Email address: vishnunarayanmishra@gmail.com (Vishnu Narayan Mishra)

doi: $10.22436 /$ jmcs.022.02.01
}

Received: 2020-04-05 Revised: 2020-04-24 Accepted: 2020-05-16 
In 1969, Fan [16] introduced the notion of a best proximity and established a classical best approximation theorem. Subsequently, many researchers have studied the best proximity point results in many ways (see in $[12,19,20,22,24,32-34,37]$ ).

The concept of b-metric space is a generalization of a metric space that was introduced by Bakhtin [6]. In 1989, Bakhtin [6] introduced the concept of b-metric space and presented the contraction mapping in b-metric spaces and later extensively used by Czerwik in $[9,10]$. The first important difference between a metric and a b-metric is that the b-metric need not be a continuous function in its two variables, see [25, Example 13]. This led to many fixed point theorems on b-metric spaces being stated, so the readers may refer to $[1-4,7,14,17,18,27-31,35,38]$ and references therein.

\section{Preliminaries}

We recall the main concepts needed to present our results.

Let $A$ and $B$ be two nonempty subsets of a metric space $(X, d)$. We denote by $A_{0}$ and $B_{0}$ the following sets:

$$
\begin{aligned}
& A_{0}=\{x \in A: d(x, y)=d(A, B) \text { for some } y \in B\}, \\
& B_{0}=\{y \in B: d(x, y)=d(A, B) \text { for some } x \in A\},
\end{aligned}
$$

where $d(A, B)=\inf \{d(x, y): x \in A, y \in B\}$. We refer to [24] for sufficient conditions that guarantee that $A_{0}$ and $B_{0}$ are nonempty.

Definition 2.1 ([10]). Let $X$ be a non-empty set and $d: X \times X \rightarrow[0, \infty)$ be a function such that for all $x, y, z \in X$ and some $s \geqslant 1$,

(1) $d(x, y)=0$ if and only if $x=y$;

(2) $d(x, y)=d(y, x)$;

(3) $d(x, y) \leqslant s[d(x, z)+d(z, y)]$.

Then $d$ is called a b-metric on $X$ and $(X, d, s)$ is called a b-metric space.

Definition $2.2([10])$. Let $(X, d, s)$ be a b-metric space. Then

(1) A sequence $x_{n}$ is called convergent to $x$, written $\lim _{n \rightarrow \infty} x_{n}=x$, if $\lim _{n \rightarrow \infty} d\left(x_{n}, x\right)=0$.

(2) A sequence $x_{n}$ is called Cauchy in $X$ if $\lim _{n, m \rightarrow \infty} d\left(x_{n}, x_{m}\right)=0$.

(3) $(X, d, s)$ is called complete if each Cauchy sequence is a convergent sequence.

Lemma 2.3 ([1]). Let $(X, d, s)$ be a b-metric space and $\lim _{n \rightarrow \infty} x_{n}=x, \lim _{n \rightarrow \infty} y_{n}=y$. Then

(a)

$$
\frac{1}{s^{2}} d(x, y) \leqslant \liminf _{n \rightarrow \infty} d\left(x_{n}, y_{n}\right) \leqslant \limsup _{n \rightarrow \infty} d\left(x_{n}, y_{n}\right) \leqslant s^{2} d(x, y) .
$$

In particular, if $x=y$, then $\lim _{n \rightarrow \infty} \mathrm{d}\left(x_{n}, y_{n}\right)=0$.

(b) For each $z \in X$,

$$
\frac{1}{s} d(x, z) \leqslant \liminf _{n \rightarrow \infty} d\left(x_{n}, z\right) \leqslant \limsup _{n \rightarrow \infty} d\left(x_{n}, z\right) \leqslant s d(x, z) .
$$

Definition 2.4 ([23]). Let $\Phi$ denote the set of all functions $\phi:[0, \infty) \rightarrow[0, \infty)$ which satisfy

(i) $\phi$ is continuous and nondecreasing;

(ii) $\phi(t)=0$ if and only if $t=0$.

Definition 2.5. Let $\Psi$ denote the set of all functions $\psi:[0, \infty) \rightarrow[0, \infty)$ which satisfy $\lim _{t \rightarrow r} \psi(t)>0$ for all $r>0$ and $\lim _{t \rightarrow 0^{+}} \psi(t)=0$. 
Definition 2.6. Let $\Theta$ denote the set of all functions $\theta:[0, \infty) \rightarrow[0, \infty)$ which satisfy

(i) $\theta$ is continuous;

(ii) $\theta(t)=0$ if and only if $t=0$.

In 1975, Dass and Gupta [11] extended the Banach contraction principle through rational expressions as follows.

Theorem $2.7([11])$. Let $(X, \mathrm{~d})$ be a complete metric space and $\mathrm{T}: \mathrm{X} \rightarrow \mathrm{X}$ be a self map of $\mathrm{X}$. If there exist $\alpha, \beta \geqslant 0$ with $\alpha+\beta<1$ satisfying

$$
d(T x, T y) \leqslant \alpha \frac{d(y, T y)[1+d(x, T x)]}{1+d(x, y)}+\beta d(x, y)
$$

for all $x, y \in X$, then $T$ has a unique fixed point in $X$.

Theorem 2.8 ([15]). Let $(X, \mathrm{~d})$ be a complete metric space and let $\mathrm{T}: \mathrm{X} \rightarrow \mathrm{X}$ be a self map of $\mathrm{X}$. If there exist $\psi, \varphi \in \Phi$ such that

$$
\varphi(d(T x, T y)) \leqslant \varphi(d(x, y))-\psi(d(x, y))
$$

for all $\mathrm{x}, \mathrm{y} \in \mathrm{X}$. Then $\mathrm{T}$ has a unique fixed point in $\mathrm{X}$.

Doric [13] extended Theorem 2.8 to a pair of self maps by replacing the monotonicity and continuity of $\psi$ by lower semi continuity, and Doric's result for the case of single self map is the following.

Theorem 2.9 ([13]). Let $(\mathrm{X}, \mathrm{d})$ be a complete metric space and let $\mathrm{T}: \mathrm{X} \rightarrow \mathrm{X}$ be a self maps of $\mathrm{X}$. If there exist $\varphi \in \Phi$ and $\psi \in \Psi$ such that

$$
\varphi(d(T x, T y)) \leqslant \varphi(M(x, y))-\psi(M(x, y))
$$

for all $\mathrm{x}, \mathrm{y} \in \mathrm{X}$, where

$$
M(x, y)=\max \left\{d(x, y), d(T x, x), d(T y, y), \frac{1}{2}[d(y, T x)+d(x, T y)]\right\} .
$$

Then $\mathrm{T}$ has a unique fixed point in $\mathrm{X}$.

Recently, Chandok et al. [8] introduced the following class of functions and used these functions to define weakly contractive maps.

$$
\Psi_{1}=\left\{\psi:[0, \infty) \rightarrow[0, \infty) \mid \text { for any sequence }\left\{x_{n}\right\} \in[0, \infty) \text { with } x_{n} \rightarrow t>0, \liminf _{n \rightarrow \infty} \psi\left(x_{n}\right)>0\right\} .
$$

In 2015, Chandok et al. [8] improved condition (2.1) of Theorem 2.9 by involving rational expressions using $\psi \in \Psi_{1}$ and proved the following.

Theorem $2.10([8])$. Let $(X, \mathrm{~d}, \leqslant)$ be a partially ordered complete metric space. Let $\mathrm{T}: \mathrm{X} \rightarrow \mathrm{X}$ be a continuous and non-decreasing mapping of X. Assume that there exist $\varphi \in \Phi$ and $\psi \in \Psi_{1}$ such that

$$
\varphi(d(T x, T y)) \leqslant \varphi(M(x, y))-\psi(N(x, y))
$$

for all $x, y \in X$, where

$$
M(x, y)=\max \left\{\frac{d(y, T y)[1+d(x, T x)]}{1+d(x, y)}, \frac{d(y, T x)[1+d(x, T y)]}{1+d(x, y)}, d(x, y)\right\}
$$

and

$$
N(x, y)=\max \left\{\frac{d(y, T y)[1+d(x, T x)]}{1+d(x, y)}, d(x, y)\right\}
$$

If there exists $x_{0} \in X$ with $x_{0} \leqslant T x_{0}$, then $T$ has a fixed point. 
Babu Gutti and Pathina [5] introduced $(\varphi, \psi)$-almost generalized weakly contractive map as follows.

Definition $2.11([5])$. Let $(X, \leqslant, d)$ be a partially ordered metric space. Let $T: X \rightarrow X$ be a self map of $X$. If there exist $\varphi \in \Phi$ and $\psi \in \Psi_{1}$ such that

$$
\varphi(d(T x, T y)) \leqslant \varphi\left(M_{1}(x, y)\right)-\psi\left(M_{2}(x, y)\right)+L N(x, y),
$$

for all $x, y \in X$, with $x \leqslant y$ where

$$
\begin{aligned}
& M_{1}(x, y)=\max \left\{\frac{d(y, T y)[1+d(x, T x)]}{1+d(x, y)}, \frac{d(y, T x)[1+d(x, T y)]}{1+d(x, y)}, d(x, y)\right\}, \\
& M_{2}(x, y)=\max \left\{\frac{d(y, T y)[1+d(x, T x)]}{1+d(x, y)}, d(x, y)\right\},
\end{aligned}
$$

and

$$
N(x, y)=\min \left\{d(x, T x), d(y, T y), d(y, T x), \frac{d(y, T x)[1+d(x, T y)]}{1+d(x, y)}\right\},
$$

then we say that $T$ is a $(\varphi, \psi)$-almost generalized weakly contractive map on $X$.

Samet et al. [36] defined the notion of $\alpha$-admissible mapping as follows.

Definition 2.12 ([36]). Let $\alpha: X \times X \rightarrow[0, \infty)$ be a function. We say that a self-mapping $T: X \rightarrow X$ is $\alpha$-admissible if

$$
x, y \in X, \quad \alpha(x, y) \geqslant 1 \Rightarrow \alpha(T x, T y) \geqslant 1 .
$$

Karapinar et al. [21] introduced the notion of triangular $\alpha$-admissible mapping as follows.

Definition 2.13 ([21]). Let $\alpha: X \times X \rightarrow[-\infty, \infty)$ be a function. We say that a self-mapping $T: X \rightarrow X$ is triangular $\alpha$-admissible if

$$
x, y \in X, \quad \alpha(x, y) \geqslant 1 \Rightarrow \alpha(T x, T y) \geqslant 1
$$

and

$$
x, y, z \in X, \quad\left\{\begin{array}{l}
\alpha(x, z) \geqslant 1 \\
\alpha(z, y) \geqslant 1
\end{array} \quad \Longrightarrow \alpha(x, y) \geqslant 1\right.
$$

Kumam et al. [26] defined the notion of triangular $\alpha$-proximal admissible as follows.

Definition 2.14 ([26]). Let $A, B$ be two nonempty subsets of a metric space $(X, d)$ and $\alpha: A \times A \rightarrow[0, \infty)$ be a function. We say that a non-self mapping $T: A \rightarrow B$ triangular $\alpha$-proximal admissible if, for all $x, y, z, x_{1}, x_{2}, u_{1}, u_{2} \in A$,

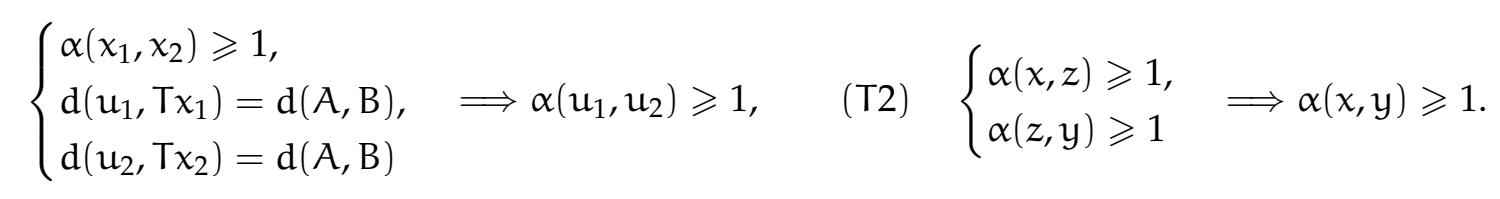

The main purpose of this paper is to introduce almost generalized $(\alpha-\psi-\varphi-\theta)$-proximal weakly contractive mapping with rational expression and to prove the best proximity point theorem for such mappings. In this way, we use the conditions (2.2), (2.3), and (2.4) of Definition 2.11 without taking $\psi \in \Psi_{1}$. Our results generalize several comparable results in the recent literature.

\section{Main results}

In this section, we first define the notion of almost generalized $(\alpha-\psi-\varphi-\theta)$-proximal weakly contractive mapping with rational expression. Then we proof best proximity point theorem for such mapping.

Definition 3.1. Let $(X, d)$ be a b-metric space. Let $A, B$ be nonempty subsets of $X$, and $T: A \rightarrow B$ be mappings. We say that $\mathrm{T}$ is an almost generalized $(\alpha-\psi-\varphi-\theta)$-proximal weakly contractive mapping for all $x_{1}, x_{2}, u_{1}, u_{2} \in A$, if there exist $\alpha: X \times X \rightarrow \mathbb{R}$ and $\psi \in \Psi, \phi \in \Phi$ and $\theta \in \Theta$ and some $L \geqslant 0$, we 
have

$$
\left\{\begin{array}{l}
d\left(u_{1}, T x_{1}\right)=d(A, B), \\
d\left(u_{2}, T x_{2}\right)=d(A, B),
\end{array} \quad \Longrightarrow \alpha\left(x_{1}, x_{2}\right) \psi\left(s^{3} d\left(u_{1}, u_{2}\right)\right) \leqslant \psi\left(M_{1}\left(x_{1}, x_{2}\right)\right)-\varphi\left(M_{2}\left(x_{1}, x_{2}\right)\right)+L \theta\left(N\left(x_{1}, x_{2}\right)\right) .\right.
$$

On the other hand,

$$
\begin{aligned}
& M_{1}\left(x_{1}, x_{2}\right)=\max \left\{\frac{d\left(x_{2}, u_{2}\right)\left\{1+d\left(x_{1}, u_{1}\right)\right\}}{1+d\left(x_{1}, x_{2}\right)}, \frac{d\left(x_{2}, u_{1}\right)\left\{1+d\left(x_{1}, u_{2}\right)\right\}}{1+d\left(x_{1}, x_{2}\right)}, d\left(x_{1}, x_{2}\right)\right\}, \\
& M_{2}\left(x_{1}, x_{2}\right)=\max \left\{\frac{d\left(x_{2}, u_{2}\right)\left\{1+d\left(x_{1}, u_{1}\right)\right\}}{1+d\left(x_{1}, x_{2}\right)}, d\left(x_{1}, x_{2}\right)\right\}, \\
& N\left(x_{1}, x_{2}\right)=\min \left\{d\left(x_{1}, u_{1}\right), d\left(x_{2}, u_{2}\right), d\left(x_{2}, u_{1}\right), \frac{d\left(x_{2}, u_{1}\right)\left\{1+d\left(x_{1}, u_{2}\right)\right\}}{1+d\left(x_{1}, x_{2}\right)}\right\} .
\end{aligned}
$$

Theorem 3.2. Let $(X, d)$ be a complete b-metric space. Let $A, B$ be nonempty closed subsets of $X$ such that $A_{0} \neq \emptyset$. Let $\mathrm{T}: \mathrm{A} \rightarrow \mathrm{B}$ be a mapping satisfying the following conditions:

(a) $\mathrm{T}$ is continuous and $\mathrm{T}\left(\mathrm{A}_{0}\right) \subseteq \mathrm{B}_{0}$;

(b) $\mathrm{T}$ is triangular $\alpha$-proximal admissible;

(c) $T$ is an almost generalized ( $\alpha-\psi-\varphi-\theta)$-proximal weakly contractive mapping on $A$;

(d) there exist elements $x_{0}, x_{1} \in A_{0}$ such that

$$
\mathrm{d}\left(\mathrm{x}_{1}, \mathrm{~T} \mathrm{x}_{0}\right)=\mathrm{d}(\mathrm{A}, \mathrm{B}) \text { and } \alpha\left(\mathrm{x}_{0}, \mathrm{x}_{1}\right) \geqslant 1 .
$$

Then $\mathrm{T}$ has a best proximity point. Further, the best proximity point is unique if for all $\mathrm{x}, \mathrm{y} \in \mathrm{A}$ such that $\mathrm{d}(\mathrm{x}, \mathrm{T} x)=\mathrm{d}(\mathrm{A}, \mathrm{B})=\mathrm{d}(\mathrm{y}, \mathrm{Ty})$, we have $\alpha(x, y) \geqslant 1$.

Proof. Let $x_{0}, x_{1} \in A_{0}$ such that

$$
\mathrm{d}\left(\mathrm{x}_{1}, \mathrm{~T} \mathrm{x}_{0}\right)=\mathrm{d}(\mathrm{A}, \mathrm{B}) \text { and } \alpha\left(\mathrm{x}_{0}, \mathrm{x}_{1}\right) \geqslant 1 .
$$

Therefore, $x_{1} \in A_{0}$, since $T\left(A_{0}\right) \subseteq B_{0}$ and $T$ is triangular $\alpha$-proximal admissible there exists $x_{2} \in A_{0}$ such that

$$
\mathrm{d}\left(\mathrm{x}_{2}, T x_{1}\right)=\mathrm{d}(\mathrm{A}, \mathrm{B}) \text { and } \alpha\left(\mathrm{x}_{1}, \mathrm{x}_{2}\right) \geqslant 1 \text {. }
$$

Continuing this process, we obtain a sequence $\left\{x_{n}\right\}$ in $A_{0}$ such that for all $n \in \mathbb{N}$,

$$
\left\{\begin{array}{l}
\alpha\left(x_{n-1}, x_{n}\right) \geqslant 1, \\
d\left(x_{n}, T x_{n-1}\right)=d(A, B), \\
d\left(x_{n+1}, T x_{n}\right)=d(A, B) .
\end{array}\right.
$$

Since, $\mathrm{T}$ is an almost generalized $(\alpha-\psi-\varphi-\theta)$-proximal weakly contractive mapping, we have

$$
\begin{aligned}
\psi\left(d\left(x_{n}, x_{n+1}\right)\right) & \leqslant \psi\left(s^{3} d\left(x_{n}, x_{n+1}\right)\right) \\
& \leqslant \alpha\left(x_{n-1}, x_{n}\right) \psi\left(s^{3} d\left(x_{n}, x_{n+1}\right)\right) \\
& \leqslant \psi\left(M_{1}\left(x_{n-1}, x_{n}\right)\right)-\varphi\left(M_{2}\left(x_{n-1}, x_{n}\right)\right)+\operatorname{L\theta }\left(N\left(x_{n-1}, x_{n}\right)\right),
\end{aligned}
$$

for all $n \in \mathbb{N}$, where

$$
\begin{aligned}
M_{1}\left(x_{n-1}, x_{n}\right) & =\max \left\{\frac{d\left(x_{n}, x_{n+1}\right)\left\{1+d\left(x_{n-1}, x_{n}\right)\right\}}{1+d\left(x_{n-1}, x_{n}\right)}, \frac{d\left(x_{n}, x_{n}\right)\left\{1+d\left(x_{n-1}, x_{n+1}\right)\right\}}{1+d\left(x_{n-1}, x_{n}\right)}, d\left(x_{n-1}, x_{n}\right)\right\}, \\
& =\max \left\{d\left(x_{n}, x_{n+1}\right), 0, d\left(x_{n-1}, x_{n}\right)\right\}, \\
& =\max \left\{d\left(x_{n}, x_{n+1}\right), d\left(x_{n-1}, x_{n}\right)\right\}, \\
M_{2}\left(x_{n-1}, x_{n}\right) & =\max \left\{\frac{d\left(x_{n}, x_{n+1}\right)\left\{1+d\left(x_{n-1}, x_{n}\right)\right\}}{1+d\left(x_{n-1}, x_{n}\right)}, d\left(x_{n-1}, x_{n}\right)\right\}, \\
& =\max \left\{d\left(x_{n}, x_{n+1}\right), d\left(x_{n-1}, x_{n}\right)\right\}, \\
N\left(x_{n-1}, x_{n}\right) & =\min \left\{d\left(x_{n-1}, x_{n}\right), d\left(x_{n}, x_{n+1}\right), d\left(x_{n}, x_{n}\right), \frac{d\left(x_{n}, x_{n}\right)\left\{1+d\left(x_{n-1}, x_{n+1}\right)\right\}}{1+d\left(x_{n-1}, x_{n}\right)}\right\}, \\
& =\min \left\{d\left(x_{n-1}, x_{n}\right), d\left(x_{n}, x_{n+1}\right), 0,0\right\}=0 .
\end{aligned}
$$


By applying the inequality (3.2) and using (3.3) we have

$$
\begin{aligned}
\psi\left(d\left(x_{n}, x_{n+1}\right)\right) & \leqslant \psi\left(s^{3} d\left(x_{n}, x_{n+1}\right)\right) \\
& \leqslant \psi\left(\max \left\{d\left(x_{n}, x_{n+1}\right), d\left(x_{n-1}, x_{n}\right)\right\}\right)-\varphi\left(\max \left\{d\left(x_{n}, x_{n+1}\right), d\left(x_{n-1}, x_{n}\right)\right\}\right), \\
& <\psi\left(\max \left\{d\left(x_{n}, x_{n+1}\right), d\left(x_{n-1}, x_{n}\right)\right\}\right) .
\end{aligned}
$$

If

$$
\max \left\{d\left(x_{n}, x_{n+1}\right), d\left(x_{n-1}, x_{n}\right)\right\}=d\left(x_{n}, x_{n+1}\right),
$$

then by (3.4), we have

$$
\psi\left(d\left(x_{n}, x_{n+1}\right)\right) \leqslant \psi\left(d\left(x_{n}, x_{n+1}\right)\right)-\varphi\left(d\left(x_{n}, x_{n+1}\right)\right),<\psi\left(d\left(x_{n}, x_{n+1}\right)\right) .
$$

which gives a contradiction. Thus,

$$
\max \left\{d\left(x_{n}, x_{n+1}\right), d\left(x_{n-1}, x_{n}\right)\right\}=d\left(x_{n-1}, x_{n}\right) .
$$

Therefore (3.4), becomes

$$
\psi\left(d\left(x_{n}, x_{n+1}\right)\right) \leqslant \psi\left(d\left(x_{n-1}, x_{n}\right)\right)-\varphi\left(d\left(x_{n-1}, x_{n}\right)\right),<\psi\left(d\left(x_{n-1}, x_{n}\right)\right) \text { for each } n \in \mathbb{N} \text {. }
$$

Since $\psi$ is a non-decreasing mapping, it follows by (3.5) that $d\left(x_{n}, x_{n+1}\right)$ is a non-increasing sequence of positive numbers which is bounded from below. So, there exists $r \geqslant 0$ such that

$$
\lim _{n \rightarrow \infty} d\left(x_{n}, x_{n+1}\right)=r .
$$

Now, we claim that $r=0$. On the contrary, assume that

$$
\lim _{n \rightarrow \infty} d\left(x_{n}, x_{n+1}\right)=r>0 .
$$

Since $\psi$ and $\varphi$ are continuous, it follows by taking limit as $n \rightarrow \infty$ in (3.5) that

$$
\psi(r)=\lim _{n \rightarrow \infty} \psi\left(d\left(x_{n}, x_{n+1}\right)\right) \leqslant \lim _{n \rightarrow \infty} \psi\left(d\left(x_{n-1}, x_{n}\right)\right)-\lim _{n \rightarrow \infty} \varphi\left(d\left(x_{n-1}, x_{n}\right)\right) \leqslant \psi(r)-\varphi(r) \leqslant \psi(r) .
$$

Therefore, $\varphi(r)=0$, and hence $r=0$. Thus, we have

$$
\lim _{n \rightarrow \infty} d\left(x_{n}, x_{n+1}\right)=0 .
$$

Now, we show that $\left\{x_{n}\right\}$ is a b-Cauchy sequence in $X$. Suppose the contrary, that is, $\left\{x_{n}\right\}$ is not a b-Cauchy sequence. Then there exists $\epsilon>0$ for which we obtain two subsequences $\left\{x_{m(i)}\right\}$ and $\left\{x_{n(i)}\right\}$ of $\left\{x_{n}\right\}$ with $n(i)>m(i) \geqslant i$ such that

$$
d\left(x_{m(i)}, x_{\mathfrak{n}(i)}\right) \geqslant \epsilon
$$

and

$$
\mathrm{d}\left(\mathrm{x}_{\mathrm{m}(\mathrm{i})}, \mathrm{x}_{\mathrm{n}(\mathrm{i})-1}\right)<\epsilon .
$$

From (3.7) and (3.8) and by using the triangular inequality in a b-metric space, we obtain that

$$
\epsilon \leqslant d\left(x_{n(i)}, x_{m(i)}\right) \leqslant s d\left(x_{n(i)}, x_{n(i)-1}\right)+s d\left(x_{n(i)-1}, x_{m(i)}\right) \leqslant s d\left(x_{n(i)}, x_{n(i)-1}\right)+s \epsilon .
$$

Taking the upper limit as $i \rightarrow \infty$ and using (3.6), we obtain

$$
\epsilon \leqslant \limsup _{i \rightarrow \infty} d\left(x_{m(i)}, x_{n(i)}\right) \leqslant s \epsilon .
$$


Also,

$$
\begin{aligned}
\epsilon & \leqslant d\left(x_{m(i)}, x_{n(i)}\right) \leqslant s d\left(x_{m(i)}, x_{n(i)+1}\right)+s d\left(x_{n(i)+1}, x_{n(i)}\right) \\
& \leqslant s^{2} d\left(x_{m(i)}, x_{n(i)}\right)+s^{2} d\left(x_{n(i)}, x_{n(i)+1}\right)+s d\left(x_{n(i)+1}, x_{n(i)}\right) \\
& \leqslant s^{2} d\left(x_{m(i)}, x_{n(i)}\right)+\left(s^{2}+s\right) d\left(x_{n(i)}, x_{n(i)+1}\right) .
\end{aligned}
$$

So, from (3.6) and (3.10), we have

$$
\frac{\epsilon}{s} \leqslant \limsup _{i \rightarrow \infty} d\left(x_{m(i)}, x_{n(i)+1}\right) \leqslant s^{2} \epsilon .
$$

Also,

$$
\begin{aligned}
\epsilon \leqslant d\left(x_{n(i)}, x_{m(i)}\right) & \leqslant s d\left(x_{n(i)}, x_{m(i)+1}\right)+s d\left(x_{m(i)+1}, x_{m(i)}\right) \\
& \leqslant s^{2} d\left(x_{n(i)}, x_{m(i)}\right)+s^{2} d\left(x_{m(i)}, x_{m(i)+1}\right)+s d\left(x_{m(i)+1}, x_{m(i)}\right) \\
& \leqslant s^{2} d\left(x_{n(i)}, x_{m(i)}\right)+\left(s^{2}+s\right) d\left(x_{m(i)}, x_{m(i)+1}\right) .
\end{aligned}
$$

So, from (3.6) and (3.8), we have

$$
\frac{\epsilon}{s} \leqslant \limsup _{i \rightarrow \infty} d\left(x_{n(i)}, x_{m(i)+1}\right) \leqslant s^{2} \epsilon
$$

Also,

$$
d\left(x_{m(i)+1}, x_{n(i)}\right) \leqslant s d\left(x_{m(i)+1}, x_{n(i)+1}\right)+s d\left(x_{n(i)+1}, x_{n(i)}\right),
$$

from (3.6) and (3.12), we have

$$
\frac{\epsilon}{s^{2}} \leqslant \limsup _{i \rightarrow \infty} d\left(x_{n(i)+1}, x_{m(i)+1}\right) .
$$

Taking (3.6), (3.8), (3.11) and (3.13) into account, we have

$$
\begin{aligned}
& \limsup _{i \rightarrow \infty} M_{1}\left(x_{m(i)}, x_{n(i)}\right)=\max \left\{\limsup _{i \rightarrow \infty} \frac{d\left(x_{n(i)}, x_{n(i)+1}\right)\left\{1+d\left(x_{m(i)}, x_{m(i)+1}\right)\right\}}{1+d\left(x_{m(i)}, x_{n(i)}\right)},\right. \\
& \limsup _{i \rightarrow \infty} \frac{d\left(x_{n(i)}, x_{m(i)+1}\right)\left\{1+d\left(x_{m(i)}, x_{n(i)+1}\right)\right\}}{1+d\left(x_{m(i)}, x_{n(i)}\right)}, \\
& \left.\limsup _{i \rightarrow \infty} d\left(x_{m(i)}, x_{n(i)}\right)\right\}, \leqslant \max \left\{d\left(0, \frac{s^{2} \epsilon\left(1+s^{2} \epsilon\right)}{1+s \epsilon}, s \epsilon\right)\right\}=s \epsilon, \\
& \limsup _{i \rightarrow \infty} M_{2}\left(x_{m(i)}, x_{n(i)}\right)=\max \left\{\limsup _{i \rightarrow \infty} \frac{d\left(x_{n(i)}, x_{n(i)+1}\right)\left\{1+d\left(x_{m(i)}, x_{m(i)+1}\right)\right\}}{1+d\left(x_{m(i)}, x_{n(i)}\right)},\right. \\
& \left.\limsup _{i \rightarrow \infty} d\left(x_{m(i)}, x_{n(i)}\right)\right\} \leqslant \max \{0, s \epsilon\}=s \epsilon, \\
& \limsup _{i \rightarrow \infty} N\left(x_{m(i)}, x_{n(i)}\right)=\min \left\{\limsup _{i \rightarrow \infty} d\left(x_{m(i)}, x_{m(i)+1}\right), \limsup _{i \rightarrow \infty} d\left(x_{n(i)}, x_{n(i)+1}\right),\right. \\
& \left.\limsup _{i \rightarrow \infty} d\left(x_{n(i)}, x_{m(i)+1}\right), \limsup _{i \rightarrow \infty} \frac{d\left(x_{n(i)}, x_{m(i)+1}\right)\left\{1+d\left(x_{m(i)}, x_{n(i)+1}\right)\right\}}{1+d\left(x_{m(i)}, x_{n(i)}\right)}\right\}, \\
& =\min \left\{0,0, s^{2} \epsilon, \frac{s^{2} \epsilon\left(1+s^{2} \epsilon\right)}{1+s \epsilon}\right\}=0 .
\end{aligned}
$$

Now, using inequality (3.1), we have

$$
\psi(s \epsilon)=\psi\left(s^{3} \cdot \frac{\epsilon}{s^{2}}\right) \leqslant \psi\left(s^{3} \limsup _{i \rightarrow \infty} d\left(x_{m(i)+1}, x_{n(i)+1}\right)\right)
$$




$$
\begin{aligned}
& =\limsup _{i \rightarrow \infty} \psi\left(s^{3} d\left(x_{m(i)+1}, x_{n(i)+1}\right)\right) \\
& \leqslant \limsup _{i \rightarrow \infty} \alpha\left(x_{m(i)}, x_{n(i)}\right) \psi\left(s^{3} d\left(x_{m(i)}, x_{n(i)}\right)\right) \\
& \leqslant \limsup _{i \rightarrow \infty}\left[\psi\left(M_{1}\left(x_{m(i)}, x_{n(i)}\right)\right)-\varphi\left(M_{2}\left(x_{m(i)}, x_{n(i)}\right)\right)+L \theta\left(N\left(x_{m(i)}, x_{n(i)}\right)\right)\right] \\
& \leqslant \psi(s \epsilon)-\varphi(s \epsilon)<\psi(s \epsilon),
\end{aligned}
$$

which is a contradiction. So we conclude that $\left\{x_{n}\right\}$ is a cauchy sequence. Since $X$ is complete b-metric space and $A$ is a closed subset of $X$, there exists $X$ in $A$ such that $x_{n} \rightarrow x$. Since $T$ is continuous, it follows that $T x_{n} \rightarrow T x$. Thus, we have

$$
\mathrm{d}\left(x_{\mathrm{n}+1}, \mathrm{~T} x_{\mathrm{n}}\right) \rightarrow \mathrm{d}(\mathrm{x}, \mathrm{T} x) .
$$

On the other hand, $d\left(x_{n+1}, T x_{n}\right)=d(A, B)$. Therefore $d(x, T x)=d(A, B)$. That is $x$ is best proximity point of $\mathrm{T}$.

As for the uniqueness of best proximity point, we now assume that there exist $x, u \in A$. Thus we have

$$
\left\{\begin{array}{l}
\alpha(x, u) \geqslant 1, \\
d(x, T x)=d(A, B), \\
d(u, T u)=d(A, B) .
\end{array}\right.
$$

Since, $T$ is an almost generalized $(\alpha-\psi-\varphi-\theta)$-proximal weakly contractive mapping, we have

$$
\begin{aligned}
& \psi(d(x, u)) \leqslant \psi\left(s^{3} d(x, u)\right) \leqslant \alpha(x, u) \psi\left(s^{3} d(x, u)\right) \leqslant \psi\left(M_{1}(x, u)\right)-\varphi\left(M_{2}(x, u)\right)+\operatorname{L\theta }(N(x, u)), \\
& \psi(d(x, u)) \leqslant \psi(d(x, u))-\varphi(d(x, u)),<\psi(d(x, u)) .
\end{aligned}
$$

This implies that $d(x, u)=0 \Rightarrow x=u$. Therefore, the best proximity point of $T$ is unique.

Theorem 3.3. Let $(X, d)$ be a complete b-metric space. Let $A, B$ be nonempty closed subsets of $X$ such that $A_{0} \neq \emptyset$. Let $\mathrm{T}: \mathrm{A} \rightarrow \mathrm{B}$ be a mapping satisfying the following conditions:

(a) $\mathrm{T}\left(\mathrm{A}_{0}\right) \subseteq \mathrm{B}_{0}$;

(b) $\mathrm{T}$ is triangular $\alpha$-proximal admissible;

(c) $T$ is an almost generalized ( $\alpha-\psi-\varphi-\theta)$-proximal weakly contractive mapping on $A$;

(d) if $\left\{x_{n}\right\}$ is a sequence in $A$ such that $\alpha\left(x_{n}, x_{n+1}\right) \geqslant 1$ and $x_{n} \rightarrow x \in A$, then $\alpha\left(x_{n}, x\right) \geqslant 1$ for all $n \in \mathbb{N}$;

(e) there exists an elements $x_{0}, x_{1} \in A_{0}$ such that

$$
\mathrm{d}\left(\mathrm{x}_{1}, \mathrm{~T} \mathrm{x}_{0}\right)=\mathrm{d}(\mathrm{A}, \mathrm{B}) \text { and } \alpha\left(\mathrm{x}_{0}, \mathrm{x}_{1}\right) \geqslant 1 .
$$

Then $\mathrm{T}$ has a best proximity point. Further, the best proximity point is unique if for all $\mathrm{x}, \mathrm{y} \in \mathrm{A}$ such that $\mathrm{d}(\mathrm{x}, \mathrm{Tx})=\mathrm{d}(\mathrm{A}, \mathrm{B})=\mathrm{d}(\mathrm{y}, \mathrm{Ty})$, we have $\alpha(x, y) \geqslant 1$.

Proof. Following the proof of Theorem 3.2, we can choose a sequence $\left\{x_{n}\right\} \subseteq A_{0}$ such that

$$
\alpha\left(x_{n+1}, x_{n}\right) \geqslant 1, \quad d\left(x_{n+1}, T x_{n}\right)=d(A, B)
$$

for all $n \in \mathbb{N}$. Also, $\left\{x_{n}\right\}$ is a Cauchy sequence and $A_{0}$ is closed, there exists an $x \in A_{0}$ such that $x_{n} \rightarrow x$, and by assumption, $\alpha\left(x_{n}, x\right) \geqslant 1$. As $T\left(A_{0}\right) \subseteq B_{0}$, there exists $u \in A_{0}$ such that

$$
d(u, T x)=d(A, B) .
$$

Therefore, we have

$$
\left\{\begin{array}{l}
\alpha\left(x_{n}, x\right) \geqslant 1, \\
d\left(x_{n+1}, T x_{n}\right)=d(A, B), \\
d(u, T x)=d(A, B) .
\end{array}\right.
$$


Since $T$ is an almost generalized $(\alpha-\psi-\varphi-\theta)$-proximal weakly contractive mapping on $A$, we have

$$
\begin{aligned}
\psi\left(d\left(x_{n+1}, u\right)\right) \leqslant \psi\left(s^{3} d\left(x_{n+1}, u\right)\right) & \leqslant \alpha\left(x_{n}, x\right) \psi\left(s^{3} d\left(x_{n+1}, u\right)\right) \\
& \leqslant \psi\left(M_{1}\left(x_{n}, x\right)\right)-\varphi\left(M_{2}\left(x_{n}, x\right)\right)+\operatorname{L\theta }\left(N\left(x_{n}, x\right)\right)
\end{aligned}
$$

for all $n \in \mathbb{N}$, where

$$
\begin{aligned}
& M_{1}\left(x_{n}, x\right)=\max \left\{\frac{d(x, u)\left\{1+d\left(x_{n}, x_{n+1}\right)\right\}}{1+d\left(x_{n}, x\right)}, \frac{d\left(x, x_{n+1}\right)\left\{1+d\left(x_{n}, u\right)\right\}}{1+d\left(x_{n}, x\right)}, d\left(x_{n}, x\right)\right\}, \\
& M_{2}\left(x_{n}, x\right)=\max \left\{\frac{d(x, u)\left\{1+d\left(x_{n}, x_{n+1}\right)\right\}}{1+d\left(x_{n}, x\right)}, d\left(x_{n}, x\right)\right\}, \\
& N\left(x_{n}, x\right)=\min \left\{d\left(x_{n}, x_{n+1}\right), d(x, u), d\left(x, x_{n+1}\right), \frac{d\left(x, x_{n+1}\right)\left\{1+d\left(x_{n}, u\right)\right\}}{1+d\left(x_{n}, x\right)}\right\} .
\end{aligned}
$$

On letting $n \rightarrow \infty$, we get

$$
\begin{aligned}
\lim _{n \rightarrow \infty} M_{1}\left(x_{n}, x\right) & =\max \{d(x, u), 0, d(u, x)\}=d(x, u), \\
\lim _{n \rightarrow \infty} M_{2}\left(x_{n}, x\right) & =\max \{d(x, u), d(u, x)\}=d(x, u), \\
\lim _{n \rightarrow \infty} N\left(x_{n}, x\right) & =\min \{d(u, x), d(x, u), 0,0\}=0 .
\end{aligned}
$$

Taking limit superior on both sides of the inequality (3.15), we have

$$
\psi(d(x, u)) \leqslant \psi\left(s^{3} d(x, u)\right) \leqslant \alpha(u, x) \psi\left(s^{3} d(x, u)\right) \leqslant \psi(d(x, u))-\varphi(d(x, u))+\text { L.0 }<\psi(d(x, u)),
$$

which is contradiction and this implies that $d(x, u)=0$ that is $u=x$. Then from (3.14), we have $d(x, T x)=$ $d(A, B)$. The proof of uniqueness is similar to the one in Theorem 3.2.

If $\mathrm{T}$ is a self mapping in Theorem 3.2 and Theorem 3.3, then we get the following.

Corollary 3.4. Let $(\mathrm{X}, \mathrm{d})$ be a complete b-metric space. Let $\mathrm{T}: \mathrm{X} \rightarrow \mathrm{X}$ be a mapping satisfying the following conditions:

(a) $T$ is continuous or if $\left\{x_{n}\right\}$ is a sequence in $X$ such that $\alpha\left(x_{n}, x_{n+1}\right) \geqslant 1$ and $x_{n} \rightarrow x \in X$, then $\alpha\left(x_{n}, x\right) \geqslant 1$ for all $\mathrm{n} \in \mathbb{N}$;

(b) $\mathrm{T}$ is triangular $\alpha$-admissible;

(c) $T$ is an almost generalized $(\alpha-\psi-\varphi-\theta)$-weakly contractive mapping that is

$$
\alpha(x, y) \psi\left(s^{3} d(T x, T y)\right) \leqslant \psi\left(M_{1}(x, y)\right)-\varphi\left(M_{2}(x, y)\right)+L \theta(N(x, y),
$$

for all $\mathrm{x}, \mathrm{y} \in \mathrm{X}$, where

$$
\begin{aligned}
& M_{1}(x, y)=\max \left\{\frac{d(y, T y)[1+d(x, T x)]}{1+d(x, y)}, \frac{d(y, T x)[1+d(x, T y)]}{1+d(x, y)}, d(x, y)\right\} \\
& M_{2}(x, y)=\max \left\{\frac{d(y, T y)[1+d(x, T x)]}{1+d(x, y)}, d(x, y)\right\}
\end{aligned}
$$

and

$$
N(x, y)=\min \left\{d(x, T x), d(y, T y), d(y, T x), \frac{d(y, T x)[1+d(x, T y)]}{1+d(x, y)}\right\}
$$

(d) there exist elements $x_{0}, x_{1} \in X$ such that 


$$
\mathrm{d}\left(\mathrm{x}_{1}, \mathrm{~T} \mathrm{x}_{0}\right)=0 \text { and } \alpha\left(\mathrm{x}_{0}, \mathrm{x}_{1}\right) \geqslant 1 \text {. }
$$

Then $T$ has a fixed point. Further, the fixed point is unique if for all $x, y \in X$ such that $d(x, T x)=0=d(y, T y)$, we have $\alpha(x, y) \geqslant 1$.

If we choose $\mathrm{L}=0$ in Theorem 3.2 and Theorem 3.3, we deduce the following corollary.

Corollary 3.5. Let $(X, d)$ be a complete b-metric space. Let $A, B$ be nonempty closed subsets of $X$ such that $A_{0} \neq \emptyset$. Let $\mathrm{T}: \mathrm{A} \rightarrow \mathrm{B}$ be a mapping and $\alpha: \mathrm{X} \times \mathrm{X} \rightarrow \mathbb{R}$ satisfying the following condition:

$$
\alpha\left(x_{1}, x_{2}\right) \psi\left(s^{3} d\left(u_{1}, u_{2}\right)\right) \leqslant \psi\left(M_{1}\left(x_{1}, x_{2}\right)\right)-\varphi\left(M_{2}\left(x_{1}, x_{2}\right)\right),
$$

where

$$
\begin{aligned}
& M_{1}\left(x_{1}, x_{2}\right)=\max \left\{\frac{d\left(x_{2}, u_{2}\right)\left\{1+d\left(x_{1}, u_{1}\right)\right\}}{1+d\left(x_{1}, x_{2}\right)}, \frac{d\left(x_{2}, u_{1}\right)\left\{1+d\left(x_{1}, u_{2}\right)\right\}}{1+d\left(x_{1}, x_{2}\right)}, d\left(x_{1}, x_{2}\right)\right\}, \\
& M_{2}\left(x_{1}, x_{2}\right)=\max \left\{\frac{d\left(x_{2}, u_{2}\right)\left\{1+d\left(x_{1}, u_{1}\right)\right\}}{1+d\left(x_{1}, x_{2}\right)}, d\left(x_{1}, x_{2}\right)\right\},
\end{aligned}
$$

for all $x_{1}, x_{2}, u_{1}, u_{2} \in A$ and $\psi, \varphi:[0, \infty) \rightarrow[0, \infty)$ are continuous functions satisfy $\psi(t)>\varphi(t)>0$ for $t>0$, $\psi(0)=\varphi(0)=0$, here $\psi$ is increasing. Assume also the following condition holds:

(a) $T$ is continuous or if $\left\{x_{n}\right\}$ is a sequence in $A_{0}$ such that $\alpha\left(x_{n}, x_{n+1}\right) \geqslant 1$ and $x_{n} \rightarrow x \in A$, then $\alpha\left(x_{n}, x\right) \geqslant 1$ for all $\mathrm{n} \in \mathbb{N}$;

(b) $\mathrm{T}$ is triangular $\alpha$-proximal admissible;

(c) $\mathrm{T}\left(\mathrm{A}_{0}\right) \subseteq \mathrm{B}_{0}$;

(d) there exist elements $x_{0}, x_{1} \in A_{0}$ such that

$$
\mathrm{d}\left(\mathrm{x}_{1}, \mathrm{~T} \mathrm{x}_{0}\right)=\mathrm{d}(\mathrm{A}, \mathrm{B}) \text { and } \alpha\left(\mathrm{x}_{0}, \mathrm{x}_{1}\right) \geqslant 1 .
$$

Then $\mathrm{T}$ has a best proximity point. Further, the best proximity point is unique if for all $\mathrm{x}, \mathrm{y} \in \mathrm{A}$ such that $\mathrm{d}(\mathrm{x}, \mathrm{Tx})=\mathrm{d}(\mathrm{A}, \mathrm{B})=\mathrm{d}(\mathrm{y}, \mathrm{Ty})$, we have $\alpha(x, y) \geqslant 1$.

From Theorem 3.2 and Theorem 3.3, if the function $\alpha: X \times X \rightarrow \mathbb{R}$ is such that $\alpha(x, y)=1$ for all $x, y \in A$, we deduce the following corollary.

Corollary 3.6. Let $(X, d)$ be a complete b-metric space. Let $A, B$ be nonempty closed subsets of $X$ such that $A_{0} \neq \emptyset$. Let $\mathrm{T}: \mathrm{A} \rightarrow \mathrm{B}$ be a mapping satisfying the following condition:

$$
\psi\left(s^{3} d\left(u_{1}, u_{2}\right)\right) \leqslant \psi\left(M_{1}\left(x_{1}, x_{2}\right)\right)-\varphi\left(M_{2}\left(x_{1}, x_{2}\right)\right)+\operatorname{L} \theta\left(N\left(x_{1}, x_{2}\right)\right),
$$

where

$$
\begin{aligned}
& M_{1}\left(x_{1}, x_{2}\right)=\max \left\{\frac{d\left(x_{2}, u_{2}\right)\left\{1+d\left(x_{1}, u_{1}\right)\right\}}{1+d\left(x_{1}, x_{2}\right)}, \frac{d\left(x_{2}, u_{1}\right)\left\{1+d\left(x_{1}, u_{2}\right)\right\}}{1+d\left(x_{1}, x_{2}\right)}, d\left(x_{1}, x_{2}\right)\right\}, \\
& M_{2}\left(x_{1}, x_{2}\right)=\max \left\{\frac{d\left(x_{2}, u_{2}\right)\left\{1+d\left(x_{1}, u_{1}\right)\right\}}{1+d\left(x_{1}, x_{2}\right)}, d\left(x_{1}, x_{2}\right)\right\}, \\
& N\left(x_{1}, x_{2}\right)=\min \left\{d\left(x_{1}, u_{1}\right), d\left(x_{2}, u_{2}\right), d\left(x_{2}, u_{1}\right), \frac{d\left(x_{2}, u_{1}\right)\left\{1+d\left(x_{1}, u_{2}\right)\right\}}{1+d\left(x_{1}, x_{2}\right)}\right\},
\end{aligned}
$$

for all $\mathrm{x}_{1}, \mathrm{x}_{2}, \mathrm{u}_{1}, \mathrm{u}_{2} \in A$ and $\psi, \varphi:[0, \infty) \rightarrow[0, \infty)$ are continuous functions satisfy $\psi(\mathrm{t})>\varphi(\mathrm{t})>0$ for $\mathrm{t}>0$, $\psi(0)=\varphi(0)=0$, here $\psi$ is increasing. Assume also the following condition holds:

(a) $T$ is continuous or if $\left\{x_{n}\right\}$ is a sequence in $A_{0}$ and $x_{n} \rightarrow x \in A$ for all $n \in \mathbb{N}$;

(b) $\mathrm{T}\left(\mathrm{A}_{0}\right) \subseteq \mathrm{B}_{0}$; 
(c) there exist elements $x_{0}, x_{1} \in A_{0}$ such that

$$
d\left(x_{1}, T x_{0}\right)=d(A, B) .
$$

Then $\mathrm{T}$ has a best proximity point. Further, the best proximity point is unique if for all $\mathrm{x}, \mathrm{y} \in \mathrm{A}$ such that $d(x, T x)=d(A, B)=d(y, T y)$.

From Corollary 3.6, if $\psi(t)=t$ and $L=0$, we deduce the following corollary.

Corollary 3.7. Let $(X, d)$ be a complete b-metric space. Let $A, B$ be nonempty closed subsets of $X$ such that $A_{0} \neq \emptyset$. Let $\mathrm{T}: \mathrm{A} \rightarrow \mathrm{B}$ be a mapping satisfying the following condition:

$$
s^{3} d\left(u_{1}, u_{2}\right) \leqslant M_{1}\left(x_{1}, x_{2}\right)-\varphi\left(M_{2}\left(x_{1}, x_{2}\right)\right)
$$

where

$$
\begin{aligned}
& M_{1}\left(x_{1}, x_{2}\right)=\max \left\{\frac{d\left(x_{2}, u_{2}\right)\left\{1+d\left(x_{1}, u_{1}\right)\right\}}{1+d\left(x_{1}, x_{2}\right)}, \frac{d\left(x_{2}, u_{1}\right)\left\{1+d\left(x_{1}, u_{2}\right)\right\}}{1+d\left(x_{1}, x_{2}\right)}, d\left(x_{1}, x_{2}\right)\right\}, \\
& M_{2}\left(x_{1}, x_{2}\right)=\max \left\{\frac{d\left(x_{2}, u_{2}\right)\left\{1+d\left(x_{1}, u_{1}\right)\right\}}{1+d\left(x_{1}, x_{2}\right)}, d\left(x_{1}, x_{2}\right)\right\},
\end{aligned}
$$

for all $x_{1}, x_{2}, u_{1}, u_{2} \in A$ and $\varphi:[0, \infty) \rightarrow[0, \infty)$ is continuous function satisfy $\varphi(t)<t$ for $t>0, \varphi(0)=0$. Assume also the following condition holds:

(a) $T$ is continuous or if $\left\{x_{n}\right\}$ is a sequence in $A_{0}$ and $x_{n} \rightarrow x \in A$ for all $n \in \mathbb{N}$;

(b) $\mathrm{T}\left(\mathrm{A}_{0}\right) \subseteq \mathrm{B}_{0}$;

(c) there exist elements $x_{0}, x_{1} \in A_{0}$ such that

$$
d\left(x_{1}, T x_{0}\right)=d(A, B) .
$$

Then $\mathrm{T}$ has a best proximity point. Further, the best proximity point is unique if for all $\mathrm{x}, \mathrm{y} \in \mathrm{A}$ such that $\mathrm{d}(x, T x)=d(A, B)=d(y, T y)$.

Example 3.8. Let $X=[0, \infty)$ be equipped with the b-metric defined by $d(x, y)=|x-y|$ with $s=2$. Let $A=\{2,3,4\}$ and $B=\{6,7,8,9,10\}$ and define the map $T: A \rightarrow B$ by

$$
T x= \begin{cases}6, & \text { if } x=4 \\ x+4, & \text { otherwise }\end{cases}
$$

Also define $\psi, \varphi, \theta:[0, \infty) \rightarrow[0, \infty)$ by $\psi(t)=t, \varphi(t)=\frac{t}{2}, \theta(t)=t$ and $\alpha(x, y)=1$. Clearly, $d(A, B)=2$, $A_{0}=\{4\}, B_{0}=\{6\}$ and $T(A) \subseteq B$.

Let $d\left(u_{1}, T x_{1}\right)=d(A, B)=2$ and $d\left(u_{2}, T x_{2}\right)=d(A, B)=2$ then $x_{1}, x_{2}, u_{1}, u_{2} \in A$. For $u_{1}=4$ and $\mathrm{u}_{2}=4$ and $\left(\mathrm{x}_{1}, \mathrm{x}_{2}\right)=(4,2)$ we have $\mathrm{M}_{1}(4,2)=2, \mathrm{M}_{2}(4,2)=2$, and $\mathrm{N}(4,2)=0$ such that

$$
\begin{aligned}
\psi\left(s^{3} \mathrm{~d}\left(\mathrm{u}_{1}, \mathrm{u}_{2}\right)\right)=\psi\left(2^{3} \mathrm{~d}(4,4)\right)=\psi(8 \cdot 0)=\psi(0)=0 & \leqslant 1 \\
& =2-1+0 \\
& =\psi(2)-\varphi(2)+\mathrm{L} \cdot 0 \\
& =\psi\left(\mathrm{M}_{1}(4,2)\right)-\varphi\left(\mathrm{M}_{2}(4,2)\right)+\mathrm{L} \cdot \theta(\mathrm{N}(4,2)) .
\end{aligned}
$$

Hence the inequality (3.1) holds true. Hence T is an almost generalized ( $\alpha-\psi-\varphi-\theta)$-proximal weakly contractive mapping and $\mathrm{T}$ satisfies all the hypotheses of Theorem 3.2 and $\mathrm{T}$ has a unique best proximity point. 
Example 3.9. Let $X=[0, \infty)$ be equipped with the b-metric defined by $d(x, y)=|x-y|$ with $s=2$. Let $A=\{3,4,5,6,7\}$ and $B=\{9,10,11,12,13\}$ and define the map $T: A \rightarrow B$ by

$$
T x= \begin{cases}9, & \text { if } x=7 \\ x+6, & \text { otherwise }\end{cases}
$$

Also define $\psi, \varphi, \theta:[0, \infty) \rightarrow[0, \infty)$ by $\psi(t)=t, \varphi(t)=\frac{t}{4}, \theta(0)=0$ if and only if $t=0$ and $\alpha(x, y)=1$. Clearly, $d(A, B)=2, A_{0}=\{7\}, B_{0}=\{9\}$ and $T\left(A_{0}\right) \subseteq B_{0}$.

Let $d\left(u_{1}, T x_{1}\right)=2$ and $d\left(u_{2}, T x_{2}\right)=2$ then $\left(u_{1}, x_{1}\right),\left(u_{2}, x_{2}\right) \in\{(7,7)(7,3)\}$. Again, assume that $d\left(u_{1}, T x_{1}\right)=d(A, B)=2$ and $d\left(u_{2}, T x_{2}\right)=d(A, B)=2$. Then $\left(x_{1}, x_{2}\right)=(7,3)$ and $u_{1}=u_{2}=7$ and we have $M_{1}(7,3)=4, M_{2}(7,3)=4$ and $N(7,3)=0$ such that

$$
\begin{aligned}
\psi\left(s^{3} \mathrm{~d}\left(\mathrm{u}_{1}, \mathrm{u}_{2}\right)\right) & =\psi\left(2^{3} \mathrm{~d}(7,7)\right) \\
& =\psi(8 \cdot 0)=\psi(0)=0 \\
& \leqslant 3 \\
& =4-1+0 \\
& =\psi(4)-\varphi(4)+\mathrm{L} \cdot \theta(0) \\
& =\psi\left(\mathrm{M}_{1}(7,3)\right)-\varphi\left(\mathrm{M}_{2}(7,3)\right)+\mathrm{L} \cdot \theta(\mathrm{N}(7,3)) .
\end{aligned}
$$

Hence the inequality (3.1) hold true. Hence T is an almost generalized ( $\alpha-\psi-\varphi-\theta)$-proximal weakly contractive mapping and $\mathrm{T}$ satisfies all the hypotheses of Theorem 3.2 and $\mathrm{T}$ has a unique best proximity point.

\section{References}

[1] A. Aghajani, M. Abbas, J. R. Roshan, Common fixed point of generalized weak contractive mappings in partially ordered b-metric spaces, Math. Slovaca, 64 (2014), 941-960. 1, 2.3

[2] A. Aghajani, R. Arab, Fixed points of $(\psi-\phi-\theta)$-contractive mappings in partially ordered b-metric spaces and application to quadratic integral equations, Fixed Point Theory Appl., 2013 (2013), 20 pages.

[3] T. V. An, L. Q. Tuyen, N. V. Dung, Stone-type theorem on b-metric spaces and applications, Topology Appl., 185/86 (2015), 50-64.

[4] H. Aydi, M.-F. Bota, E. Karapinar, S. Mitrović, A fixed point theorem for set-valued quasi-contractions in b-metric spaces, Fixed Point Theory Appl., 2012 (2012), 8 pages. 1

[5] V. R. Babu Gutti, S. K. Pathina, Fixed Points Of $(\psi, \varphi)$-almost generalized weakly contractive maps with rational expressions in partially ordered metric spaces, J. Int. Math. Virtual Inst., 7 (2017), 69-83. 2, 2.11

[6] I. A. Bakhtin, The contraction mapping principle in almost metric spaces, Funct. Anal., 30 (1989), 26-37. 1

[7] M. Boriceanu, M. Bota, A. Petrusel, Multivalued fractals in b-metric spaces, Cent. Eur. J. Math., 8 (2010), 367-377. 1

[8] S. Chandok, B. S. Choudhury, N. Metiya, Fixed point results in ordered metric spaces for rational type expressions with auxiliary functions, J. Egyptian Math. Soc., 23 (2015), 95-101. 2, 2.10

[9] S. Czerwik, Contraction mappings in b-metric spaces, Acta Math. Inform. Univ. Ostraviensis, 1 (1993), 5-11. 1

[10] S. Czerwik, Nonlinear set-valued contraction mappings in b-metric spaces, Atti Sem. Mat. Fis. Univ. Modena, 46 (1998), 263-276. 1, 2.1, 2.2

[11] B. K. Dass, S. Gupta, An extension of Banach contraction principle through rational expressions, Indian J. Pure Appl. Math., 6 (1975), 1455-1458. 2, 2.7

[12] M. Dinarvand, Fixed points of almost contractive type mappings in partially ordered b-metric spaces and applications to quadratic integral equations, Facta Univ. Ser. Math. Inform., 31 (2016), 775-800. 1

[13] D. Doric, Common fixed point for generalized ( $\psi, \varphi)$-weak contraction, Appl. Math. Lett., 22 (2009), 1896-1900. 2, 2.9

[14] N. V. Dung, N. T. T. Ly, V. D. Thinh, N. T. Hieu, Suzuki-type fixed point theorems for two maps in metric-type spaces, J. Nonlinear Anal. Optim., 4 (2013), 17-29. 1

[15] P. N. Dutta, B. S. Choudhury, A generalisation of contraction principle in metric spaces, Fixed Point Theory Appl., 2008 (2008), 8 pages. 2.8

[16] K. Fan, Extensions of two fixed point theorems of FE Browder, Math. Z., 112 (1969), 234-240. 1

[17] H. P. Huang, S. Y. Xu, Fixed point theorems of contractive mappings in cone b-metric spaces and applications, Fixed Point Theory Appl., 2013 (2013), 8 pages. 1

[18] N. Hussain, V. Parvaneh, J. R. Roshan, Z. Kadelburg, Fixed points of cyclic weakly ( $\psi, \varphi, L, A, B)$-contractive mappings in ordered b-metric spaces with applications, Fixed Point Theory Appl., 2013 (2013), 18 pages. 1 
[19] H. Işık, H. Aydi, N. Mlaiki, S. Radenovic̀, Best Proximity Point Results for Geraghty Type ZProximal Contractions with an Application, Axioms, 8 (2019), 12 pages. 1

[20] H. Iş1k, M. S. Sezen, C. Vetro, $\varphi$-Best proximity point theorems and applications to variational inequality problems, J. Fixed Point Theory Appl., 19 (2017), 3177-3189. 1

[21] E. Karapinar, P. Kumam, P. Salimi, On $\alpha$ - -Meir-Keeler contractive mappings, Fixed Point Theory Appl., 2013 (2013), 12 pages. 2, 2.13

[22] E. Karapinar, V. Pragadeeswarar, M. Marudai, Best proximity point for generalized proximal weak contractions in complete metric space, J. Appl. Math., 2014 (2014), 6 pages. 1

[23] M. S. Khan, M. Swaleh, S. Sessa, Fixed point theorems by altering distances between the points, Bull. Austral. Math. Soc., 30 (1984), 1-9. 2.4

[24] W. A. Kirk, S. Reich, P. Veeramani, Proximinal retracts and best proximity pair theorems, Numer. Funct. Anal. Optim., 24 (2003), 851-862. 1, 2

[25] P. Kumam, N. V. Dung, V. T. L. Hang, Some equivalences between cone b-metric spaces and b-metric spaces, Abstr. Appl. Anal., 2013 (2013), 8 pages. 1

[26] P. Kumam, P. Salimi, C. Vetro, Best proximity point results for modified $\alpha$-proximal C-contraction mappings, Fixed Point Theory Appl., 2014 (2014), 16 pages. 2, 2.14

[27] X.-L. Liu, M. Zhou, L. N. Mishra, V. N. Mishra, B. Damjanović, Common fixed point theorem of six self-mappings in Menger spaces using (CLR $\mathrm{ST}$ ) property, Open Math., 16 (2018), 1423-1434. 1

[28] L. N. Mishra, On existence and behavior of solutions to some nonlinear integral equations with applications, Ph.D. Thesis (National Institute of Technology), India, (2017).

[29] L. N. Mishra, K. Jyoti, A. Rani Vandana, Fixed point theorems with digital contractions image processing, Nonlinear Sci. Lett. A, 9 (2018), 104-115.

[30] L. N. Mishra, S. K. Tiwari, V. N. Mishra, Fixed point theorems for generalized weakly S-contractive mappings in partial metric spaces, J. Appl. Anal. Comput., 5 (2015), 600-612.

[31] L. N. Mishra, S. K. Tiwari, V. N. Mishra, I. A. Khan, Unique Fixed Point Theorems for Generalized Contractive Mappings in Partial Metric Spaces, J. Funct. Spaces, 2015 (2015), 8 pages. 1

[32] H. K. Nashine, P. Kumam, C. Vetro, Best proximity point theorems for rational proximal contractions, Fixed Point Theory Appl., 2013 (2013), 11 pages. 1

[33] V. Pragadeeswarar, M. Marudai, Best proximity points for generalized proximal weak contractions satisfying rational expression on ordered metric spaces, Abstr. Appl. Anal., 2015 (2015), 6 pages.

[34] V. S. Raj, A best proximity point theorem for weakly contractive non-self-mappings, Nonlinear Anal., 74 (2011), $4804-$ 4808. 1

[35] J. R. Roshan, V. Parvaneh, S. Sedghi, N. Shobkolaei, W. Shatanawi, Common fixed points of almost generalized $(\psi, \phi)$ scontractive mappings in ordered b-metric spaces, Fixed Point Theory Appl., 2013 (2013), 23 pages. 1

[36] B. Samet, C. Vetro, P. Vetro, Fixed point theorem for $\alpha-\psi$-contractive type mappings, Nonlinear Anal., 75 (2012), 2154-2165. 2, 2.12

[37] W. Sanhan, C. Mongkolkeha, P. Kumam, Generalized proximal $\phi$-contraction mappings and Best proximity points, Abstr. Appl. Anal., 2012 (2012), 19 pages. 1

[38] W. Shatanawi, A. Pitea, R. Lazović, Contraction conditions using comparison functions on b-metric spaces, Fixed Point Theory Appl., 2014 (2014), 11 pages. 1 